\section{Implantação da telessaúde na atenção à saúde indígena no Brasil}

\author{
Implementation of telemedicine in indigenous \\ people's healthcare in Brazil
}

\author{
Implantación de la telesalud en la atención a la \\ salud indígena en Brasil
}

\author{
${ }^{1}$ Ministério da Saúde, \\ Brasília, Brasil. \\ 2 Programa de Pós-graduação \\ em Saúde Coletiva, \\ Universidade de Brasília \\ Brasília, Brasil \\ 3 Programa de Pós- \\ graduação em Assistência \\ Farmacêutica, Universidade \\ Federal de Santa Catarina, \\ Florianópolis, Brasil. \\ Correspondência \\ M. D. A. Scherer \\ Programa de Pós-graduação \\ em Saúde Coletiva \\ Universidade de Brasília. \\ SCLN 406 Asa Norte, Brasília, \\ DF 70847-510, Brasil. \\ magscherer@hotmail.com
}

\begin{abstract}
The Brazilian National Telemedicine Program in indigenous healthcare assists health professionals working in remote areas in strengthening the healthcare provided to indigenous populations. The current study aimed to analyze the implementation of the National Telemedicine Program in indigenous people's health, from the perspective of health administrators. This was a qualitative exploratory descriptive study using document analysis and open-ended interviews with 10 administrators. Content analysis resulted in two categories: the program's process implementation and the potential of telemedicine for indigenous people's healthcare. The results emphasize the importance of dialogue between all institutions involved and the construction of a democratic forum for evaluating this process and related decision-making: resumption of the program's implementation and subsequently its expansion and improvement of the available resources and the search for other applicable strategies for indigenous people's health. A broad discussion on this topic is recommended that involves the indigenous people's strategies for social control.
\end{abstract}

Telemedicine; Continuing Education; Health of Indigenous People
Zaira Zambelli Taveira 1

Magda Duarte dos Anjos Scherer 2 Eliana Elisabeth Diehl 3

\section{Resumo}

No Brasil, o Programa Telessaúde Brasil Redes na atenção à saúde indígena é uma das estratégias para auxiliar na fixação de profissionais em áreas remotas e fortalecer a assistência à população indígena. O estudo objetivou analisar a implantação do programa voltado para a saúde indígena, com base na visão dos gestores. É um estudo exploratório descritivo com abordagem qualitativa (análise documental e entrevistas não estruturadas com dez gestores) e a análise de conteúdo resultou em duas categorias: $o$ processo de implantação do programa e as potencialidades da telessaúde na atenção à saúde indígena. Os resultados recomendam o diálogo entre as instituições envolvidas e a construção de um espaço democrático visando à avaliação desse processo e à tomada de decisão sobre o caminho a seguir: retomada da implantação $e$ consequente expansão da telessaúde, a melhoria de dispositivos já disponíveis elou a busca de outras estratégias viáveis para o contexto da saúde indígena. Além disso, sugere-se que o tema seja objeto de amplo debate nas instâncias indígenas de controle social.

Telemedicina; Educação Continuada; Saúde de Populações Indígenas 


\section{Introdução}

A Educação Permanente em Saúde é estratégia potente para o desenvolvimento das competências profissionais necessárias para a atenção primária à saúde ${ }^{1}$ mas difícil de desencadear em áreas remotas, ainda mais em contextos interculturais e interétnicos, como o da atenção à saúde indígena. A formação dos profissionais deve contemplar aspectos socioantropológicos, linguísticos e epidemiológicos, tendo em vista os diversos contextos étnicos, mas na prática as capacitações dos profissionais da saúde indígena têm sido caracterizadas por descontinuidade e desconsideração às especificidades socioculturais 2,3,4,5.

As Tecnologias de Informação e Comunicação (TICs) podem potencializar a Educação Permanente em Saúde 6,7. No Brasil, em 2009, o Programa Telessaúde Brasil Redes na atenção à saúde indígena foi implantado como uma das estratégias para enfrentar a rotatividade e a falta de preparação dos profissionais para atuação em contextos interculturais, bem como a dificuldade de efetivar a Educação Permanente em Saúde nos Distritos Sanitários Especiais Indígenas (DSEI) 8. O programa favoreceria a assistência à população indígena pela tele-educação, teleconsultoria e telediagnóstico ${ }^{2,9,10}$.

A rede de serviços dos DSEI tem como porta de entrada as unidades básicas integradas aos serviços de referência do Sistema Único de Saúde (SUS). As Casas de Saúde do Índio (CASAI) abrigam pacientes durante $\mathrm{o}$ tratamento fora das aldeias.

O presente trabalho analisou o processo de implantação do Programa Telessaúde na atenção à saúde indígena, com base na visão dos gestores.

\section{Método}

O estudo descritivo exploratório qualitativo 11 foi realizado em 2012 nos DSEI Alto Rio Negro (Pólo Base Yauaretê) e Parintins (Pólo Base Umirituba), Amazonas, Brasil, onde se localizavam os dois únicos pontos do Programa Telessaúde para a atenção à saúde indígena.

Os dados foram obtidos por pesquisa documental e entrevistas não estruturadas, e submetidos à análise temática 11. Participaram dez gestores, sendo dois de cada DSEI e seis do Núcleo de Telessaúde do Amazonas, responsáveis pela condução da implantação dos pontos.

O projeto foi aprovado pelo Comitê de Ética em Pesquisa da Faculdade de Ciências da Saúde da Universidade de Brasília (no 97/2012).

\section{Resultados e discussão}

Os projetos de implantação de pontos de telessaúde (únicos documentos encontrados: Programa Nacional de Telessaúde em Apoio à Atenção Primária, Ministério da Saúde. Projeto Telessaúde no apoio à saúde das populações indígenas e de fronteira no Estado do Amazonas. Projeto estadual de telessaúde voltado ao Subsistema de Saúde Indígena e das populações de fronteira no Estado do Amazonas. Brasília: Ministério da Saúde; s.d. Programa Nacional de Telessaúde em Apoio à Atenção Primária, Ministério da Saúde. Projeto Telessaúde no apoio à saúde das populações indígenas no Estado do Amazonas. Projeto estadual de telessaúde voltado ao Subsistema de Saúde Indígena. Brasília: Ministério da Saúde; 2010) objetivavam melhorar a qualidade da atenção primária à saúde para populações localizadas em áreas remotas e culturalmente diversas, considerando o diferenciado processo saúde/doença e a medicina tradicional das populações indígenas. Os serviços abrangiam teleassistência, tele-educação e segunda opinião formativa.

Em Yauaretê, os equipamentos foram instalados no Pelotão Especial de Fronteira, escolha justificada pela ausência de espaço na unidade de saúde do Pólo Base, de fornecimento constante de energia elétrica e da presença diária de profissional de saúde no Pelotão. A escolha foi considerada problemática por causa do histórico de confrontos entre os povos indígenas e o exército.

Durante a pesquisa, os pontos estavam inativos e os entrevistados indicaram pouco tempo de funcionamento, dificultado principalmente pela rotatividade dos profissionais, infraestrutura inadequada e dificuldades no uso da tecnologia.

A contratação dos profissionais era realizada mediante parcerias com organizações indígenas e prefeituras municipais ${ }^{12}$. Havia rotatividade dos profissionais relacionada ao intervalo das contratações, resultando em irregularidade na oferta dos serviços e na perda daqueles com experiência em saúde indígena e já capacitados no uso das ferramentas do programa. A precarização dos vínculos de trabalho guarda relação com a descontinuidade nos processos educativos 13 , e gera a necessidade de capacitar novos profissionais, o que tem um custo elevado para o sistema de saúde 14 . Além disso, dificulta a manutenção dos equipamentos e o planejamento da Educação Permanente em Saúde.

A infraestrutura inadequada caracterizava-se pela existência de equipamentos danificados e falta de uso (devido à ausência dos profissionais em área por um período prolongado), além da falta de energia elétrica. As dificuldades de funcionamento também foram associadas à localiza- 
ção geográfica, como observado em comunidades indígenas do Canadá 15. No Pólo Base de Umirituba, o transporte de equipamentos é aéreo e fluvial, sendo uma das preocupações frequentes entre os países pesquisados pela Organização Mundial da Saúde (OMS), por elevar o custo da telessaúde 16.

O treinamento inadequado ou inexistente é um fator negativamente associado à implantação das TICs 17. A falta de habilidade com os equipamentos foi relacionada ao não uso da ferramenta e à danificação dos mesmos (qualificados como sensíveis).

Os gestores enfatizaram a necessidade de articulação interinstitucional na gestão do programa, evidenciando o peso da dimensão política no processo de implantação 16. Houve articulação entre algumas instituições no planejamento para a implantação dos pontos, mas faltou clareza na definição dos papéis institucionais fora e dentro do programa, especialmente no que se referia à resolução dos entraves existentes no seu funcionamento e ampliação. Em momento algum foi mencionada a participação dos profissionais da saúde indígena, da Fundação Nacional do Índio e dos Conselhos de Saúde Indígena no planejamento da implantação do programa.

A preparação para atuação em contexto intercultural deveria ser premissa para o ingresso dos profissionais no subsistema 2 , porém isto nunca ocorreu sistematicamente 4,5,18. O Programa Telessaúde poderia ser uma ferramenta para melhorar essa situação. Os gestores, provavelmente em função do pouco tempo de funcionamento do programa, discorreram mais sobre as potencialidades e benefícios do que a telessaúde traria para ações de tele-educação. Afirmaram que a equipe poderia receber capacitação para doenças prevalentes na localidade, diminuindo os índices de mortalidade e morbidade, evitando o deslocamento dos indígenas para fora das comunidades. $\mathrm{O}$ agente indígena de saúde foi o grupo mais citado para as ações de tele-educação, cuja formação é estratégia estruturante do subsistema 2.

A redução dos encaminhamentos dos indígenas para serviços de referência contribuiria para a diminuição dos problemas sociais (na medida em que o indígena não precisaria se deslocar para a CASAI) e para a racionalização dos gastos 19, uma vez que o transporte aéreo era o mais indicado e, também, pelo elevado custo de permanência na CASAI (em função da deficiência do sistema regulador da média e alta complexidades). A superlotação e as condições precárias da CASAI, associadas à presença de diferentes etnias, exacerbam desavenças históricas existentes, apontadas como geradoras de dificuldades à permanência dos indígenas.

\section{Considerações finais}

Os gestores indicaram a retomada da implantação do programa, mas os resultados permitem questionar se de fato houve implantação e se a telessaúde é estratégia promissora na saúde indígena para a expansão da Educação Permanente em Saúde e a qualificação da atenção, já que as dificuldades resultaram no não funcionamento do programa.

Recomenda-se o diálogo entre as instituições envolvidas e a construção de um espaço democrático, com a participação dos indígenas e dos profissionais de saúde, visando à avaliação desse processo e à tomada de decisão sobre o caminho a seguir. A retomada da implantação da telessaúde, a melhoria de dispositivos já disponíveis (como a radiocomunicação) e/ou a busca de outras estratégias viáveis para o contexto da saúde indígena são algumas possibilidades. Sugere-se que o tema seja objeto de amplo debate nas instâncias indígenas de controle social. 


\section{Resumen}

En Brasil, el Programa Telesalud en la atención a la salud indígena es una de las estrategias utilizadas para la ubicación de profesionales en áreas remotas y el fortalecimiento de la asistencia a la población indígena. El estudio analizó la implantación del Programa en la salud indígena, en base a la visión de gestores. El estudio fue cualitativo exploratorio y descriptivo (análisis de documentos y entrevistas no estructuradas con 10 gestores), y el análisis de contenido se tradujo en dos categorías: el proceso de implantación del programa y el potencial de la telesalud en la atención a la salud indígena. Los resultados recomiendan el diálogo entre las instituciones involucradas y la construcción de un espacio democrático que evalúe el proceso y la toma de decisión sobre el camino a seguir: la reanudación de la implementación y la expansión del programa, la mejora de los dispositivos ya disponibles y/o la búsqueda de otras estrategias viables para el contexto de la salud indígena. Además, se sugiere que el tema sea debatido en los espacios indígenas de control social.

Telemedicina; Educación Continua; Salud de Poblaciones Indígenas

\section{Colaboradores}

Z. Z. Taveira trabalhou na concepção do projeto, na coleta e análise dos dados e na redação do artigo, e aprovou a versão a ser publicada. M. D. A. Scherer contribuiu na concepção do projeto, na análise dos dados, na revisão e aprovação final da versão a ser publicada. E. E. Diehl participou na análise dos dados, na revisão e na aprovação da versão a ser publicada.

\section{Agradecimentos}

À Capes pela Bolsas de Estágio Pós-doutoral no Exterior de M. D. A. Scherer e de Estágio Sênior no Exterior de E. E. Dihel.

\section{Referências}

1. Scherer MDA, Pires D, Jean R. A construção da interdisciplinaridade no trabalho da equipe de saúde da família. Ciênc Saúde Coletiva 2013; 18 : 3203-12.

2. Fundação Nacional de Saúde. Política Nacional de Atenção à Saúde dos Povos Indígenas. 2a Ed. Brasília: Fundação Nacional de Saúde; 2002.

3. Fundação Nacional de Saúde. Relatório final da 4a Conferência Nacional de Saúde Indígena. Brasília: Fundação Nacional de Saúde; 2007.
4. Diehl EE, Langdon EJ, Dias-Scopel RP. Contribuição dos agentes indígenas de saúde na atenção diferenciada à saúde dos povos indígenas brasileiros. Cad Saúde Pública 2012; 28:819-31.

5. Pontes AL, Stauffer A, Garnelo L. Profissionalização indígena no campo da saúde: desafios para a formação técnica de Agentes Indígenas de Saúde. In: Garnelo L, Pontes AL, organizadores. Saúde indígena: uma introdução ao tema. Brasília: Secretaria de Educação Continuada, Alfabetização, Diversidade e Inclusão, Ministério da Educação; 2012. p. 265-88. (Coleção Educação para Todos). 
6. Campos FC, Haddad AE, Wen CL, Alkmim MBM. Telessaúde em apoio à atenção primária à saúde no Brasil. In: Santos AF, Souza C, Alves HJ, Santos SF, organizadores. Telessaúde: um instrumento de suporte assistencial e educação permanente. Belo Horizonte: Universidade Federal de Minas Gerais; 2006. p. 59-74.

7. Santos AF, Souza C, Melo MCB, Santos SF. Structuring of the Brazilian Public Health System and the development of telehealth activities in Brazil. Latin American Journal of Telehealth 2009; 1:5-38.

8. Fundação Nacional de Saúde. Diagnóstico situacional do Subsistema de Saúde Indígena; relatório inicial (revisado). http://www.funasa.gov.br/internet/ arquivos/vigisus/vigModSsi_DiagnosticoSSI.pdf (acessado em 06/Jun/2011).

9. Elliott G, Smith AC, Bensink ME, Brown C, Stewart C, Perry C, et al. The feasibility of a communitybased mobile telehealth screening service for Aboriginal and Torres Strait Islander children in Australia. Telemed J E Health 2010; 16:950-6.

10. Mahapatra AK1, Mishra SK, Kapoor L, Singh IP. Critical issues in medical education and the implications for telemedicine technology. Telemed J E Health 2009; 15:592-6.

11. Minayo MCS. O desafio do conhecimento: pesquisa qualitativa em saúde. 12a Ed. São Paulo: Editora Hucitec; 2010.

12. Garnelo L. Política de saúde indígena no Brasil: notas sobre as tendências atuais do processo de implantação do subsistema de atenção à saúde. In: Garnelo L, Pontes AL, organizadores. Saúde indígena: uma introdução ao tema. Brasília: Secretaria de Educação Continuada, Alfabetização, Diversidade e Inclusão, Ministério da Educação; 2012. p. 18-59. (Coleção Educação para Todos).
13. Faria MGA. Telessaúde Brasil - Núcleo Rio de Janeiro: a educação permanente no trabalho de enfermeiros da atenção básica [Dissertação de Mestrado]. Rio de Janeiro: Universidade do Estado do Rio de Janeiro; 2010.

14. Andrade CSGC. Agentes comunitários de saúde e os desafios da educação permanente: reflexões sobre a experiência do Programa Telessaúde Brasil - Núcleo Rio de Janeiro [Dissertação de Mestrado]. Rio de Janeiro: Universidade do Estado do Rio de Janeiro; 2011.

15. Muttitt S, Vigneault R, Loewen L. Integrating telehealth into aboriginal. Healthcare: the Canadian experience. Int J Circumpolar Health 2004; 63:401-14.

16. World Health Organization. Telemedicine: opportunities and developments in Member States: report on the second global survey on eHealth. Geneva: World Health Organization; 2009. (Global Observatory for eHealth Series, 2).

17. Gagnon MP, Desmartis M, Labrecque M, Car J, Pagliari C, Pluye P, et al. Systematic review of factors influencing the adoption of information and communication technologies by healthcare professionals. J Med Syst 2012; 36:241-77.

18. Langdon EJ, Diehl EE, Wiik FB, Dias-Scopel R. A participação dos agentes indígenas de saúde nos serviços de atenção à saúde: a experiência em Santa Catarina, Brasil. Cad Saúde Pública 2006; 22:2637-46.

19. Costa CA, Souza PE, Wen CL, Böhm GM, Mota MEC. Telehealth in the Amazon: development, results and perspectives. Latin American Journal of Telehealth 2009; 1:170-83.

Recebido em 18/Fev/2014

Versão final reapresentada em 15/Abr/2014

Aprovado em 05/Mai/2014 